\title{
Evolution of pore structure during microwave freeze-drying of Chinese yam
}

\author{
Liuliu Duan, Xu Duan*, Guangyue Ren \\ (Food and Biology Engineering College, Henan University of Science \& Technology, Luoyang 471023, China)
}

\begin{abstract}
In order to reduce the energy consumption of freeze drying (FD), microwave freeze drying (MFD) can be used to dry Chinese yam. Porosity is a critical factor influencing transport mechanism, and can be considered as an important index to reflect the changes of structure of MFD foods. In this study, the changes of pore structure during the process of MFD Chinese yam were investigated by SEM and mercury porosimetry. The results showed that some closed pores could transform to open pores in drying process, and the open porosity showed a rising trend throughout the drying process. The pore size distribution range was about $10 \mathrm{~nm}$ to $10^{6} \mathrm{~nm}$ throughout the drying process. In the early stage of drying, the pore size was mainly in the range of $10-10^{4} \mathrm{~nm}$, and then the pore size and the number of pores reduced. In the middle and late drying stages, the size of large pores increased again.
\end{abstract}

Keywords: microstructure, microwave freeze drying, porosity

DOI: $10.25165 /$ j.ijabe. 20181106.4250

Citation: Duan L L, Duan X, Ren G Y. Evolution of pore structure during microwave freeze-drying of Chinese yam. Int J Agric \& Biol Eng, 2018; 11(6): 208-212.

\section{Introduction}

The Chinese yam (Dioscorea opposita) is a very popular edible and medicinal plant in China. Especially iron yam (D. opposita Thunb) is not only rich in nutrients including protein, amino acids, starches, sugars and vitamins, but also has numerous active constituents present in its tubers, such as flavonoids, polysaccharides and steroidal saponins. Many studies have confirmed that polysaccharides in Chinese yam can enhance immunity and lower blood sugar, and have pharmacological functions ${ }^{[1,2]}$. However, fresh Chinese yam is difficult to store and easy to deteriorate during storage. Therefore, it is also processed into various kinds of products when it is consumed as a vegetable. Iron yam slices dried by hot-air drying (AD) are the common products in markets and Chinese medicine shops.

Nevertheless, the relatively long drying time, high temperatures, and high velocities of drying air flow all constitute serious disadvantages for the method of AD. This leads to degradation of biological components and far-reaching changes in the quality of the product ${ }^{[3]}$. The present demand for high-quality dehydrated products in the market requires dried foods to be maintained at a very high level to preserve the nutritional and organoleptical properties of the initial fresh product ${ }^{[4]}$. Freeze-drying (FD) produces the highest quality food products among all drying methods, but it is considered the most expensive operation for manufacturing dehydrated products owing to high energy consumption and high costs of both operation and maintenance ${ }^{[5]}$. Recently, microwave freeze drying (MFD) has

\section{Received date: 2018-03-21 Accepted date: 2018-07-23}

Biographies: Liuliu Duan, Master candidate, research interests: processing of agricultural products. Email: liu786129855@qq.com; Guangyue Ren, PhD, Professor, research interests: processing of agricultural products. Email: guangyueyao@163.com.

*Corresponding author: $\mathrm{Xu}$ Duan, PhD, Professor, research interests: processing of agricultural products. Food and Biology Engineering College, Henan University of Science \& Technology, Luoyang 471023, China. Tel: +86-13653872870, Email: duanxu_dx@163.com. been developed to reduce the FD energy consumption ${ }^{[6]}$. It was proved that the drying time could be reduced greatly compared to $\mathrm{FD}$, which resulted in the potential to replace conventional FD. MFD can yield almost the same product quality as freeze-drying ${ }^{[7]}$. Jiang et al. ${ }^{[7]}$ compared the effects of different drying methods on the quality of dried banana. The results showed that the rehydration and sensory indices of MFD products were better than those of products produced by other methods ${ }^{[8]}$.

It is well known that FD foods are characterized by such characteristics as low bulk density and high porosity. In fact, the knowledge of porous structure is important in characterization of the textural properties of foods ${ }^{[9]}$. The knowledge of the porous structure of dehydrated materials may help to predict water diffusivity in foods and modeling of several mass transfer applications in foods. Aprajeeta et al. ${ }^{[10]}$ studied changes along with simultaneous heat and mass transport during potato hot air drying process, and they found that porosity also played a critical role in mass transfer. Ramachandran et al. ${ }^{[11]}$ indicated that the porosity of the sample was more critical than its apparent size. Thus it can be seen that porosity is a critical factor influencing transport mechanism, and can be considered as an important index to reflect the changes of structure of food materials.

Although both MFD and FD belong to sublimation drying methods, there are different temperature profiles between MFD and FD process, which leads to different quantities of sublimated water, resulting in various product structure and texture ${ }^{[12]}$. Sablani et al. ${ }^{[13]}$ found that the shelf temperature significantly affected the apparent density and porosity of selected food materials during FD process. Especially in the second drying phase of FD, some open pores could change to closed pores because of the collapse of the wall of the matrix that surrounded the ice crystal ${ }^{[13]}$.

Compared with FD, the drying rate of MFD is much faster and the pore structure formation is also complex. To our knowledge, there is no report about the porosity formation and change behavior during MFD process. The complexity of the pore formation mechanism needs to be further studied with diversified food materials and a wider variation of processing conditions in order to 
develop a more unified theory of pore formation. Therefore, the objective of this study was to explore the evolution characteristics of the pore structure during the MFD process of Chinese yam.

\section{Materials and methods}

\subsection{Sample preparation and drying experiments}

Fresh Chinese yam (D. opposita Thunb.) was purchased from a local market in Luoyang, China. The Chinese yams were washed, peeled and sliced to different thickness $(2,4$ and $6 \mathrm{~mm})$. The slices $(1 \mathrm{~kg})$ were then placed in a polypropylene tray and frozen at $-25^{\circ} \mathrm{C}$ for at least $8 \mathrm{~h}$. In the experiment, three microwave power loading levels $(1.5,2.7$, and $4.4 \mathrm{~W})$ were applied. All MFD processes were carried out at $100 \mathrm{~Pa}$ cavity pressure and $-40^{\circ} \mathrm{C}$ cold trap temperature. All of the experiments above were repeated twice and the average of results was used for analyzed.

\subsection{Drying equipment}

The laboratory-scale microwave freeze dryer was developed by authors ${ }^{[14]}$. As shown in Figure 1, an independent polypropylene drying cavity was set up in a rectangle resonant cavity, which could effectively avoid the corona discharge at the vacuum condition. The pressure of the drying cavity was operated at a range of $10 \mathrm{~Pa}$ to $30 \mathrm{kPa}$ (absolute pressure). The power of microwave could be adjusted continually. The core temperature of materials was detected by the optic fiber sensor.

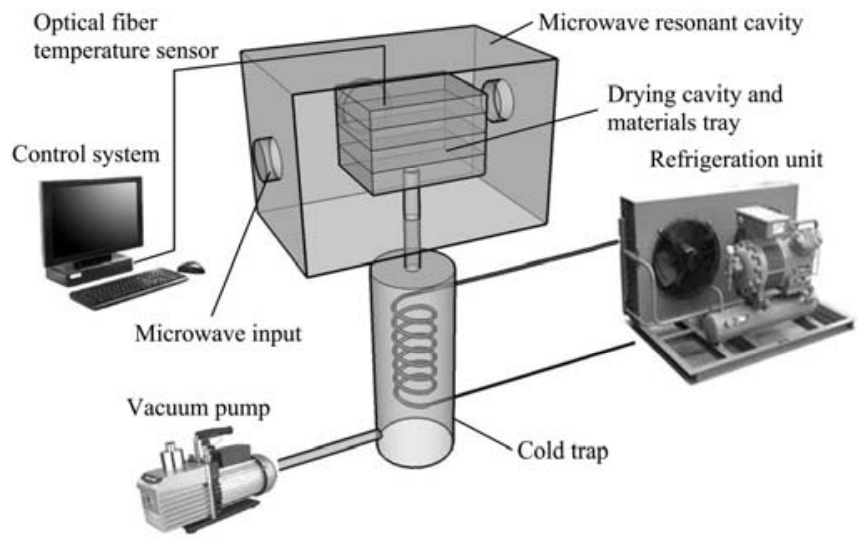

Figure 1 The schematic diagram of microwave freeze dryer

\subsection{Analysis of samples}

\subsubsection{Total porosity $(\varepsilon)$}

The porosity of sample was determined by the pycnometer. At 30 minute intervals during the drying processes samples were taken out, crushed it to below $0.15 \mathrm{~mm}$, soaked in $20^{\circ} \mathrm{C}$ n-hexane, and then held on a pycnometer for $30 \mathrm{~min}$. Weighing was performed on a digital balance. The tests were performed in triplicate. The porosity of samples under different drying conditions was calculated according to the Equation (1) and (2) as follows:

$$
\begin{gathered}
\rho_{s}=\frac{m_{s} \rho}{m_{s}+m_{1}-m_{2}} \\
\varepsilon=\left(1-\frac{m_{s}}{V \rho_{s}}\right) \times 100 \%
\end{gathered}
$$

where, $m_{s}$ is the weight of samples, $\mathrm{g}$; $\rho$ is the density of $\mathrm{n}$-hexane at $20^{\circ} \mathrm{C}, \mathrm{g} / \mathrm{cm}^{3} ; m_{1}$ is the weight of the pycnometer filled with $\mathrm{n}$-hexane, $\mathrm{g} ; m_{2}$ is the mass of the pycnometer filled with samples and n-hexane, $\mathrm{g} ; V$ is the volume of samples, $\mathrm{cm}^{3} ; \rho_{s}$ is the true density of the samples, $\mathrm{g} / \mathrm{cm}^{3}$.

\subsubsection{Pore volume $(v)$}

The pore volume was calculated according to the Equation (3) as follows:

$$
v=\varepsilon V
$$

where, $\varepsilon$ is the porosity of samples; $V$ is the total volume of samples, $\mathrm{cm}^{3}$.

\subsubsection{Open porosity $\left(\varepsilon_{0}\right)$}

The pores that are interconnected within the material or connected to the surface of the material are called open pores, and the pores that are divided into independent pores inside the material are called closed pores. The sample was placed in a $50 \mathrm{~mL}$ beaker and immersed by n-hexane. After two hours, the saturated sample was taken out, wiped out the free n-hexane on the surface of the sample, and then weighed with an electronic balance. Each experiment was performed in triplicate. The open porosity was calculated according to Equation (4).

$$
\varepsilon_{0}=\frac{m_{2}-m_{1}}{\rho V} \times 100 \%
$$

where, $m_{1}$ is the weight of the sample before immersion, $\mathrm{g} ; m_{2}$ is the weight of immersed sample, $\mathrm{g} ; \rho$ is the density of n-hexane, $\mathrm{g} / \mathrm{cm}^{3} ; V$ is the sample volume, $\mathrm{cm}^{3}$.

\subsubsection{Closed porosity $\left(\varepsilon_{1}\right)$}

The closed porosity was calculated according to the Equation (5) as follows:

$$
\varepsilon_{1}=\varepsilon-\varepsilon_{0}
$$

where, $\varepsilon$ is the total porosity of the sample, $\% ; \varepsilon_{0}$ is the open porosity, \%.

\subsubsection{Scanning electron microscopy (SEM)}

Chinese yam slices (thickness of $4 \mathrm{~mm}$ ) were microwave freeze dried at microwave power of $2.7 \mathrm{~W}$ and pressure of $100 \mathrm{~Pa}$ conditions, and samples were taken out for SEM at $30 \mathrm{~min}$ intervals during the drying processes. The selected sample was placed on conductive adhesive tabs, mounted on a scanning electron microscope (S4800, Hitachi, Japan) tubs and coated with gold-palladium in a sputter coater. The internal pore morphology of the coated samples was observed at an accelerating voltage of $4 \mathrm{kV}$.

2.3.6 Pore size and pore distribution

Chinese yam slices (thickness of $4 \mathrm{~mm}$ ) were microwave freeze dried at microwave power of $2.7 \mathrm{~W}$ and pressure of $100 \mathrm{~Pa}$ conditions, and sampled at $30 \mathrm{~min}, 60 \mathrm{~min}, 90 \mathrm{~min}$, and $120 \mathrm{~min}$ during the drying process. The selected sample was analyzed by a Mercury Porosimetry (AutoPore IV 9500, Micromeritics instrument corporation, USA). The AutoPore IV 9500 provided full-scale resolution in the following pressure ranges: low pressure (4-345 kPa), and high pressure (0.1-414 MPa). For testing, the sample was placed in the dilatometer, and then cleansed of absorbed and/or adsorbed gases by degassing in a vacuum $(4 \mathrm{kPa})$. The dilatometer, still under vacuum, was then filled with mercury. The pressure in the dilatometer was built up gradually to a final pressure of $414 \mathrm{MPa}$ for the high-pressure tests and $345 \mathrm{kPa}$ for low-pressure tests, in approximately $15 \mathrm{~min}$. The mean pore diameter was given from the AutoPore software.

\section{Results and discussion}

\subsection{Effect of different slice thickness on the porous structure of MFD Chinese yam}

Figure 2 shows the changes of pore volume and porosity of Chinese yam with different slice thickness during MFD process. It was found that the porosity of samples gradually increased with the drying progress, which implied that the porous structure of Chinese yam gradually formed during the drying process. The pore volume of samples with different slice thickness showed an 
upward tendency, but there was a slight decrease trend in the early stage of drying process. The possible reason of porosity decrease in the drying time of $0-60 \mathrm{~min}$ was contributed to the volume shrinkage of samples at this stage. The volume of Chinese yam continued to shrink, which gradually slowed down the increase of the pore volume, resulting in a decrease of the porosity of samples. The porosity increased continuously in the drying time of 60$90 \mathrm{~min}$, and remained stable after $90 \mathrm{~min}$. On the other hand, the volume shrinkage of samples after $90 \mathrm{~min}$ had no significant effect on the pore volume and the porosity.
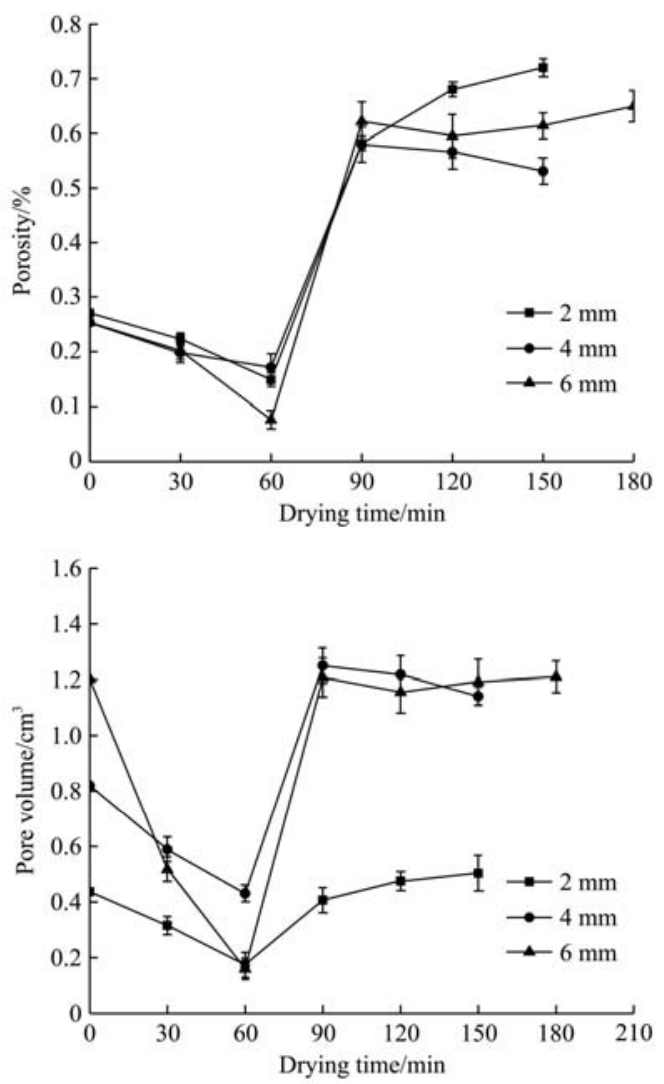

Figure 2 Porosities and Pore volumes of MFD Chinese yam with different slice thickness

It was showed that slice thickness could impact porosity. The possible reason was that the moisture content of the material gradually decreased with the progress of drying. Under the condition of low water content, the porous network skeleton of the material was relatively easy to be glass state so as to reduce the shrinkage of the volume, which was helpful to pores formation.

As was shown in Figure 3, open porosity of samples increased with the drying time. The open porosity was the smallest when the slice thickness was $4 \mathrm{~mm}$. This phenomenon indicated that open pores formed continuously during MFD process. It was illustrated that thick Chinese yam slices could lead to relatively high open porosity. Figure 4 shows the change of closed porosity of Chinese yam of different slice thickness during MFD process. Its change tendency was the same as total porosity shown in Figure 2. The closed porosity of samples dropped quickly in the initial drying stage $(0-60 \mathrm{~min})$, then rose fast in the later period (60$90 \mathrm{~min}$ ) and finally almost kept constant in the end stage of drying. Compared with Figure 3, it could be concluded that lots of closed pores constantly transformed into open pores during the drying process. Moreover, Figure 4 illustrated that thick Chinese yam slices could lead to relatively high closed porosity. The possible reason was that the temperature distribution was not very uniform when the slices were thick, then collapse occurred in some over-heat parts of samples ${ }^{[15]}$, resulting increase of closed pores.

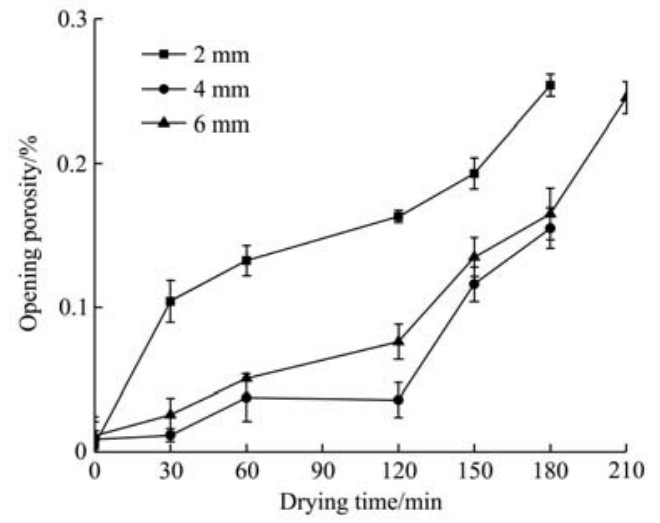

Figure 3 Open porosity variation of MFD Chinese yam with different slice thickness

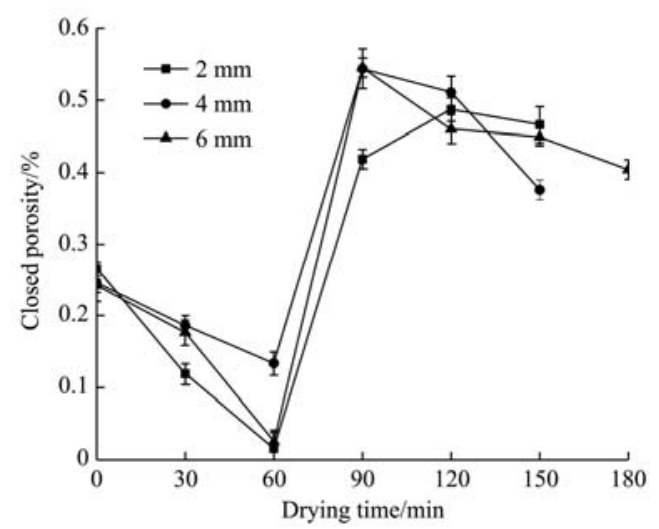

Figure 4 Close porosity variation of MFD Chinese yam with different slice thickness

\subsection{Effects of microwave power on the porous structure of MFD Chinese yam}

As shown in Figure 5, high microwave power led to relatively high porosity, and the lowest porosity was obtained when microwave power was $1.5 \mathrm{~W}$. High microwave could accelerate water sublimation and improve porosity of products, but extreme high microwave power also could lead to great deformation and collapse of materials. As a result, in order to obtain products with high porosity, relatively lower microwave power should be applied. Especially at the end stage of drying, lower microwave power could lead to a higher porosity of samples and good product quality. Moreover, the pore volume exhibited a similar change tendency to the porosity, which indicated that porosity and pore volume were both affected by volumetric shrinkage.

As shown in Figure 6, high open porosity could be obtained under high microwave power, and the lowest open porosity was obtained when microwave power was $1.5 \mathrm{~W}$. Besides, from Figure 7, it was observed that the closed porosity declined slowly at the beginning of drying, which implied that the formation of closed pores were greatly affected by volume shrinkage at the initial stage of drying, and the pore volume decreased due to volume shrinkage (Figure 7). Simultaneously, some closed pores transformed into open pores, resulting in a decrease of closed porosity. After $90 \mathrm{~min}$ of the drying process, a large number of closed pores transformed into open pores, resulting in a rapid increase of open porosity and a slight decrease of closed pore porosity. Generally, in order to obtain a structure with more open porosity, a relatively high microwave can be applied. However, 
extreme high microwave power could lead to deterioration of product quality ${ }^{[16]}$. Therefore, a suitable microwave power should be applied.
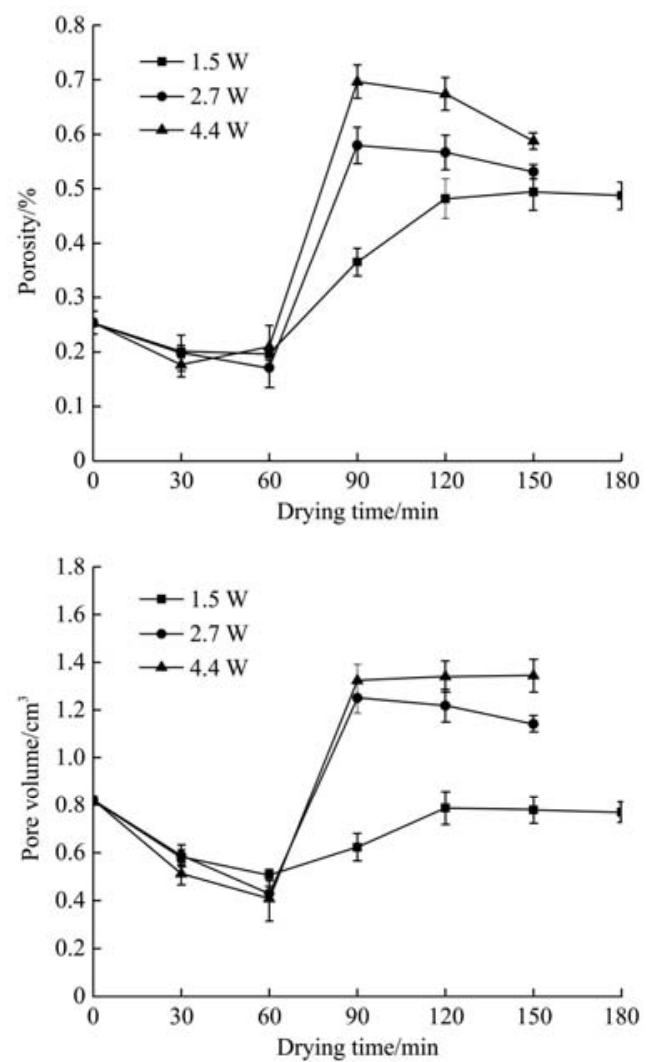

Figure 5 Porosity and pore volume variation of MFD Chinese yam under different microwave power

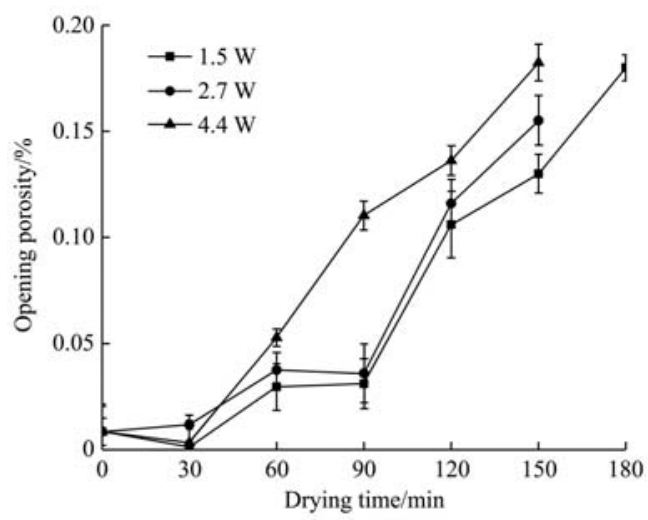

Figure 6 Open porosity variation of MFD Chinese yam under different microwave power

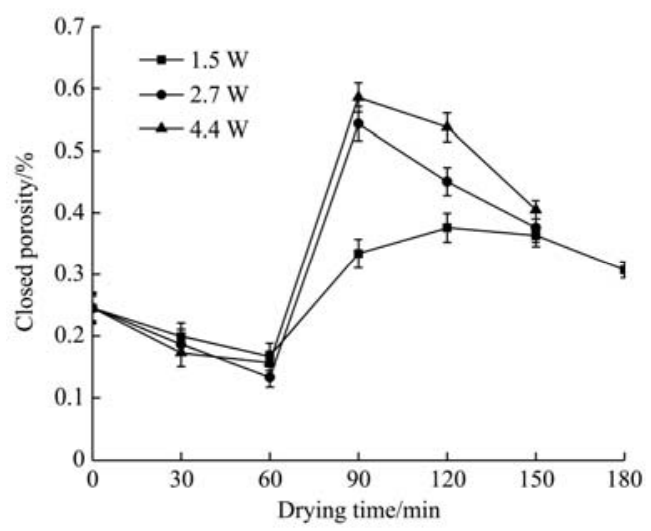

Figure 7 Closed porosity variation of MFD Chinese yam under different microwave power

\subsection{Pore structure evolution of Chinese yam during MFD} process

It can be seen from Figure 8 that the pore diameter of the sample was in the range of $10^{3}-8 \times 10^{3} \mathrm{~nm}$ at the initial drying stage. The pore diameter of products was in the range of $3 \times 10^{2}-6 \times 10^{3} \mathrm{~nm}$ in the second drying stage, and the pore diameter was in the range of $5 \times 10^{3}-10^{4} \mathrm{~nm}$ in the end drying stage. It was shown that the pore diameter changed significantly during the whole drying process, and the shrinkage deformation was significant in the early stage of drying. The possible reason was that the drying rate in the early stage of drying (0-60 min) was fast and shrinkage of the material was serious, and some large pores was compressed into small ones, so that the pore volume showed a downward trend in this stage. On the other hand, after $60 \mathrm{~min}$ of drying, shrinkage of materials became weak, so that the pore size regains gradual increase.

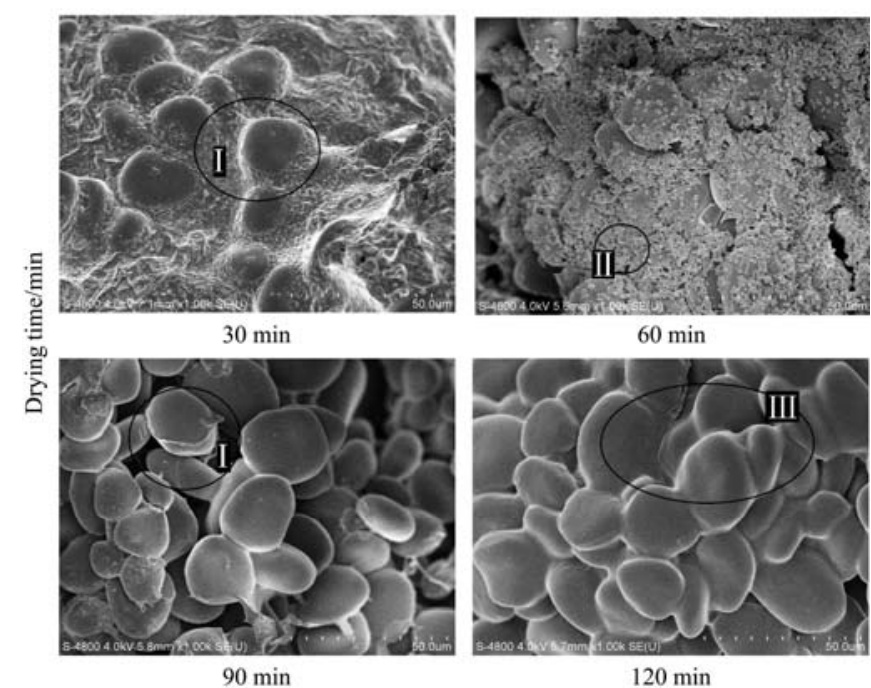

Figure 8 SEM images of MFD Chinese yam at different drying times $(\times 1.00 \mathrm{~K})$

According to Figure 8 and reports of Luo et al ${ }^{[17]}$, three kinds of pore diameters were found in the whole drying process, including large closed pore type I, small closed pore type II and open pore type III. In the early stage of drying, lots of type I pores formed, and then with rapid sublimation of moisture in Chinese yam more type II pores gradually grew up. During this period, type II pores increased significantly, and some of type II pores also transformed into type I pores. When drying proceeded to the middle stage $(90 \mathrm{~min})$, the type II pores were completely transformed into type I pores. At the end drying stage, some channels between various pores were connected due to water transfer, and then some closed pores transformed into open pores, which were called type III. In the middle and end drying period, the proportion of type III pores increased. It suggested that in the MFD Chinese yam process, a large number of closed pores formed firstly, followed by lots of closed pores opened by moisture transfer, and then the open pores increased in the end of drying stage.

In order to further determine the pore size changes of MFD Chinese yam during drying process, a more intuitive analysis was conducted by the mercury intrusion method, and the results were shown in Figure 9.

The peak area of Figure 9 represented the amount of mercury intrusion, and the amount of mercury intrusion represented the amount of pores. It can be observed from Figure 9 that the pore size distribution range is about $10-10^{6} \mathrm{~nm}$ throughout the drying process. In the initial drying stage, most of pores were in the 
range of $10-10^{4} \mathrm{~nm}$. In the second drying stage, the pore size of samples slightly decreased. The large pores above $10^{5} \mathrm{~nm}$ almost completely disappeared. In the middle and end drying period, the number of large and small pores increased significantly, and the large pores above $10^{5} \mathrm{~nm}$ reappeared. Although the pore size increased again, the proportion of pores in the range of $10^{2}-10^{4} \mathrm{~nm}$ still occupied the vast majority. The mercury withdrawal curve had an obvious hysteresis and did not return to zero, indicating that there were a lot of "bottleneck pores" in the dried Chinese yam ${ }^{[18]}$, which implied that the sample contained a large number of open pores. This result was consistent with the analysis of the SEM images. When the drying time was $30 \mathrm{~min}, 60 \mathrm{~min}, 90 \mathrm{~min}$ and $120 \mathrm{~min}$, the maximum mercury inlets were $0.507,0.262,0.448$ and $0.512 \mathrm{~mL} / \mathrm{g}$, respectively. It indicated that the volume shrinkage had an impact on the formation of pores during the first $60 \mathrm{~min}$ of drying time, and the number of pores decreased with the effect of volume shrinkage. After 60 min drying time, the volume of the material had nearly no more shrunk, and the numbers of pores began to rise again, which exhibited a similar analysis to the Figure 2 and 5.

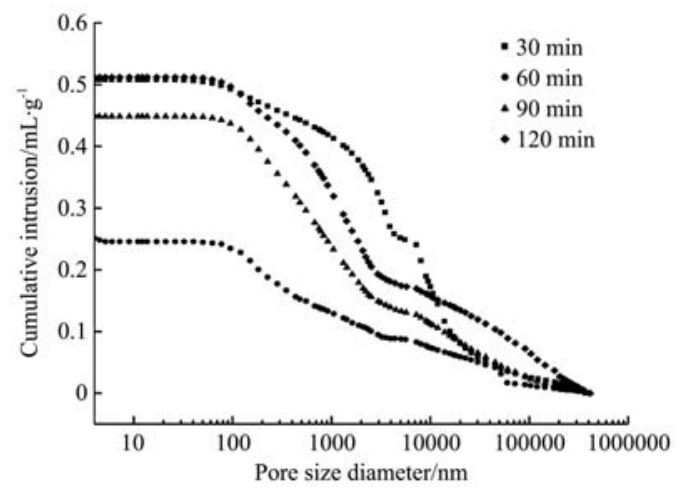

Figure 9 Results of mercury intrusion test of MFD Chinese yam at different drying times

According to the reports of $\mathrm{Lin}^{[19]}$, as water was removed, some original closed pore would open, and sometimes some original small pores were interconnected to form large pores. It was observed from Figure 9 that the mercury injection curves at 90 min was similar to the mercury injection curves at $120 \mathrm{~min}$, and the only difference was that the amount of mercury at 120 min was more than that at $90 \mathrm{~min}$. It also was observed from Figure 8 that new pores hardly appeared after $90 \mathrm{~min}$ of drying, and lots of pores merged with others to become the "bottleneck pores". Moreover, open pores gradually increased, thus the pore volume and the total porosity keep increasing. The possible reason was that the mutual integration of pores increased the number of open pores, so that the mercury injection increased.

\section{Conclusions}

The volume shrinkage of MFD Chinese yam mainly occurred in the early stage of drying, and was little in the later stage of drying. The volume shrinkage in 0-60 min of drying time had a great influence on the formation of pores. At this stage, the total porosity showed a decreasing trend, and the pore volume and pore diameter were also affected by the volume shrinkage of samples. Moreover, closed pores formed firstly during MFD process, and then some closed pores could transform into open pores. The pore size distribution of Chinese yam during MFD process was in the range of $10 \mathrm{~nm}$ to $10^{6} \mathrm{~nm}$. In the initial stage of drying, the pore size of samples slightly decreased, and large pores above $10^{5} \mathrm{~nm}$ almost completely disappeared. And then the pore diameter increased again after 60 min of drying. In the middle and end drying period, the number of both large and small pores increased significantly, and the large pores above $10^{5} \mathrm{~nm}$ reappeared. Throughout the drying process, lots of closed pores continued to transform into open pores, and the total porosity keep increasing.

\section{Acknowledgements}

This project was financially supported by the National Natural Science Foundation of China (No. 31671907) and the Natural Science Foundation of Henan Province (No. 182300410062). The authors also thank the support of the Program for Science and Technology Innovation Team in Universities of Henan Province (No. 16IRTSTHN009).

\section{[References]}

[1] Chen Y. Pharmacological effects and product development of yam. Sichuan Food and Fermentation, 2015; 51(1): 60-62

[2] Zhou Y, Guo H, Zhou J. Study on main nutrients in iron Dioscorea opposita Thunb . Food and Nutrition in China, 2011; 17(3): 69-71.

[3] Luo D L, Liu J, Liu Y H, Ren G Y. Drying characteristics and mathematical model of ultrasound assisted hot-air drying of carrots. Int J Agric \& Biol Eng, 2015; 8(4): 124-132.

[4] Duan X, Yang X, Ren G et al. Technical aspects in freeze drying of foods. Drying Technology, 2015(11).

[5] Huang L L, Min Z, Mujumdar A S, et al. Studies on decreasing energy consumption for a freeze-drying process of apple slices. Drying Technology, 2009; 27(9): 938-946.

[6] Qian G, Qi Z, Cui Z. Reducing energy consumption of carrot slices dehydration by combined vacuum microwave and freeze drying. Transactions of the Chinese Society of Agricultural Engineering, 2011; 27(6): 387-392.

[7] Qian Z, Zhang G C, Gang M, Liu Y. Freeze and microwave vacuum combination drying technique for sea cucumber. Int J Agric \& Biol Eng, 2012.

[8] Jiang H, Zhang M, Mujumdar A S, Lim R X. Comparison of drying characteristic and uniformity of banana cubes dried by pulse-spouted microwave vacuum drying, freeze drying and microwave freeze drying. Journal of the Science of Food \& Agriculture, 2014; 94(9): 1827.

[9] Huang L L, Qiao F, Fang C F. Studies on the microstructure and quality of iron yam slices during combined freeze drying and microwave vacuum drying. Journal of Food Processing \& Preservation, 2015; 39(6): 2152-2160.

[10] Aprajeeta J, Gopirajah R, Anandharamakrishnan C. Shrinkage and porosity effects on heat and mass transfer during potato drying. Journal of Food Engineering, 2015; 144: 119-128.

[11] Lai F C. Effects of Porosity on the Performance of EHD-Enhanced Drying. Drying Technology, 2010; 28(12): 1477-1483.

[12] Duan X, Liu W, Ren G Y, Liu W C, Liu Y H. Comparative study on the effects and efficiencies of three sublimation drying methods for mushrooms. Int J Agric \& Biol Eng, 2015; 8(1): 91-97.

[13] Sablani S S, Rahman M S. Pore formation in selected foods as a function of shelf temperature during freeze drying. Drying Technology, 2002; 20(7): 1379-1391

[14] Duan X, Ren G Y, Zhu W X. Microwave freeze drying of apple slices based on the dielectric properties. Drying Technology, 2012; 30(5): 535-541.

[15] Dai H M, Guo W, Cheng Y D, Jin Y Z. Three-dimensional temperature distribution of the packaged foods with different shapes during microwave heating. Science and Technology of Food Industry, 2015; 36(13): 82-86.

[16] Pereira N R, Jr A M, Ahrné L M. Effect of microwave power, air velocity and temperature on the final drying of osmotically dehydrated bananas. Journal of Food Engineering, 2007; 81(1): 79-87.

[17] Luo L Q, Liu B, An F W. The pore structure of expanded graphite and its characterization, chemical Industry and Engineering Progress. Chemical Industry and Engineering Progress, 2017; 36(2): 611-617.

[18] Gu Y F, Wang Z F, Qi L L. Study on porous structure difference of soft coal and hard coal based on mercury intrusion method. Coal Science and Technology, 2016; 44(4): 64-67.

[19] Lin X F, Yin Y S, Li Z Q. Analysis of pore structure of biomass coke by mercury pressure method. Journal of Engineering Thermophysics, 2006; 27(S2): 187-190. 